\title{
Hematological indices in congenital male hypogonadism and the effects of testosterone replacement therapy: a retrospective study
}

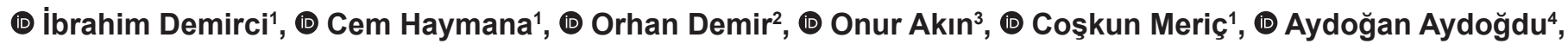 \\ (1) Alper Sönmez ${ }^{1}$
}

1 University of Health Sciences Turkey, Gülhane Training and Research Hospital, Clinic of Endocrinology and Metabolism, Ankara, Turkey

2Osmaniye Public Hospital, Clinic of Endocrinology and Metabolism, Osmaniye, Turkey

3University of Health Sciences Turkey, Gülhane Training and Research Hospital, Clinic of Pediatric Endocrinology, Ankara, Turkey ${ }^{4}$ Live Hospital, Clinic of Endocrinology and Metabolism, Ankara, Turkey

\section{Date submitted:}

16.03.2021

Date accepted:

02.05.2021

\section{Online publication date:} 15.09.2021

\section{Corresponding Author:}

İbrahim Demirci, M.D., University of Health Sciences Turkey, Gülhane

Training and Research Hospital, Clinic of Endocrinology and Metabolism,

Ankara, Turkey

dr.idemirci@gmail.com

ORCID:

orcid.org/ 0000-0003-0498-3115

Keywords: Hypogonadism, testosterone, cardiometabolic risk, NLR, PLR

\begin{abstract}
Aims: Mean platelet volume (MPV), platelet-to-lymphocyte ratio (PLR), and neutrophil-tolymphocyte ratio (NLR) are surrogate markers of adverse cardiovascular (CV) outcomes. The aim of this study was to investigate the platelet count, MPV level, PLR, NLR, and the effect of testosterone replacement therapy (TRT) on these parameters in patients with congenital hypogonadotropic hypogonadism $(\mathrm{CHH})$.

Methods: Young male patients with $\mathrm{CHH}$ and healthy controls were recruited from the outpatient setting. Blood pressures, height, weight, waist circumference (WC), and blood tests including triglyceride (TG) level, insulin, homeostatic model assessment-insulin resistance (HOMA-IR), PLR, and NLR were calculated between $\mathrm{CHH}$ patients and controls and following TRT.

Results: Sixty-six patients with $\mathrm{CHH}$ (mean age: $21.5 \pm 2.0$ years) and 67 healthy controls (mean age: $21.9 \pm 1.3$ years) were included in the study. $\mathrm{CHH}$ patients had higher platelet count (262.9 \pm 50.7 vs $232.1 \pm 49.2, p=0.001)$, MPV level $(8.63 \pm 0.87$ vs $8.16 \pm 0.92, p=0.004)$, PLR (114.01 \pm 25.68 vs $100.59 \pm 25.49, p=0.003)$, WC $(86.8 \pm 9.1$ vs $83.7 \pm 7.9, p=0.04), T G(96.0$ vs 80.0 , $\mathrm{p}=0.02)$, insulin (9.41 vs $6.92, \mathrm{p}<0.001)$, and HOMA-IR ( 1.95 vs $1.47, \mathrm{p}<0.001)$ than healthy controls. NLR was similar in both groups. After TRT, CHH patients showed significant elevations in body mass index, WC, total testosterone, and HOMA-IR and reduction in high density lipoprotein cholesterol level; however, platelet count, MPV, PLR, and NLR indices remained unchanged. Total testosterone level was also correlated with platelet count, MPV, and PLR.
\end{abstract}

Conclusions: This study showed higher platelet count and surrogate markers of $\mathrm{CV}$ risk such as MPV and PLR in patients with CHH. However, short time TRT did not show any effect on these indices.

\section{Introduction}

Patients with hypogonadism present with clinical findings of low testosterone levels such as low libido, loss of energy, depression, and muscle atrophy. In addition to fertility defects, the prevalence of type 2 diabetes, hypertension, and dyslipidemia is increased in patients with hypogonadism $(1,2)$.
Recent studies have also showed a relationship between low testosterone level and atherosclerosis, coronary artery disease, or cardiovascular (CV) events $(3,4)$. Inflammation and thrombosis have important roles in the pathogenesis of atherosclerosis and CV diseases (CVD) (5). Testosterone deficiency is associated with metabolic profiles that are known to be associated with atherosclerosis such as high glucose, low 
density lipoprotein cholesterol (LDL-Chol) and pro-inflammatory cytokine levels and low high density lipoprotein cholesterol (HDL-Chol) and anti-inflammatory cytokine levels (6). However, the mechanism of increased atherosclerosis and CVD risk in patients with hypogonadism is still unknown.

The hematological indices, mean platelet volume (MPV), platelet-to-lymphocyte ratio (PLR), and neutrophil-to-lymphocyte ratio (NLR) are surrogate markers of adverse CV outcomes. MPV is a new CV risk factor, which is considered to be a simple marker of platelet size and activation (7). Metabolically and enzymatically more active larger platelets have higher thrombotic potential. Elevated MPV level is reported in coronary artery disease and in many other diseases such as hypertension, diabetes and hypercholesterolemia $(8,9)$. Platelets have a critical role in emerging of atherosclerosis. There is an association between elevated blood platelet counts and CVD. Also, previous studies reported an association between lymphopenia and CVD. The ratio of the platelet to lymphocyte count, PLR is a prognostic marker in different types of CVD (10-12). Similarly, NLR is also a novel marker of inflammation, and is reported to be associated with adverse cardiac outcomes $(11,13)$.

All of these hematological indices are inexpensive, easy to obtain, and widely available markers of adverse cardiac outcomes. However, there are limited data about the levels of these parameters in patients with congenital hypogonadism. It is also unknown whether the testosterone replacement therapy (TRT) affects these hematological parameters. Therefore, in this retrospective study, we aimed to investigate the platelet count, MPV level, PLR, and NLR in patients with hypogonadism and also study the effect of TRT on these indices.

\section{Methods}

\section{Study Design and Patient Selection}

This retrospective analysis was performed using the database of an endocrinology unit of a tertiary hospital. Male patients with the diagnosis of congenital hypogonadotropic hypogonadism $(\mathrm{CHH})$, who were 18 years old or over and never received TRT or human chorionic gonadotropin, were identified and included in the analyses. Patients under 18 years old or who had pituitary mass lesions in magnetic resonance imaging or had laboratory values consistent with panhypopituitarism or who had any other chronic metabolic disorder and/or organ dysfunction were excluded. The stage of pubertal development (Tanner's stage), serum level of total testosterone, follicle stimulating hormone $(\mathrm{FSH})$ and luteinizing hormone $(\mathrm{LH})$ were obtained from the database. The subjects in the control group were selected from the previous studies performed on this database $(14,15)$. Control subjects did not have chronic disorders and were not taking any drug treatment. Also, none of the patients or control subjects had any signs of infection or had a history of any medication known to influence platelet function at least 2 weeks (e.g., acetylsalicylate, antiepileptics, or heparin) before study initiation. The Local Ethical Committee of University of Health Sciences Turkey Gülhane Faculty of Medicine approved the study (code: 16.06.2016, 50687569-1491-440-16/1648-1562).

\section{Definitions}

The methods for the assessment of anthropometric and laboratory variables in the database were as follows; height, weight, and waist circumference (WC) were measured while wearing underwear. After exhalation, WC was measured from the line on the iliac crest parallel to the ground. Body mass index (BMI) was computed as the ratio of weight to the square of height $\left(\mathrm{kg} / \mathrm{m}^{2}\right)$. Arterial blood pressures were measured two times by a mercury column sphygmomanometer with appropriate-size cuff after a resting period of at least $5 \mathrm{~min}$. The average of the measurements was recorded as the systolic and diastolic blood pressures of the patients and controls. The anthropometric measurements and laboratory values at three and/or six-month follow-up of treatment were retrieved from the database.

The venous blood samples of the patient and control groups were collected between 08:00 and 09:00 h after an overnight fasting and were stored at $-80{ }^{\circ} \mathrm{C}$ after centrifugation for 15 min. Complete blood count was obtained using the Olympus AU-2700 autoanalyzer $(\mathrm{GmbH})$. Fasting plasma glucose, total cholesterol, triglyceride (TG), and HDL-Chol level were measured by the enzymatic colorimetric method, and serum basal insulin, total testosterone, FSH and $\mathrm{LH}$ level were measured by the chemiluminescence method. LDL-Chol level was calculated with the Friedewald formula. For the enzymatic calorimetric analyses Olympus AU-2700 autoanalyzer (Hamburg, Germany), and chemiluminence analyses UniCel DxI 800 Access Immunoassay System (Miami, FL, USA) was used. Insulin sensitivity was calculated by the homeostatic model assessment-insulin resistance (HOMA-IR) using the following formula: HOMA-IR=[insulin $(\mathrm{mU} / \mathrm{mL}) \times$ glucose $(\mathrm{mg} / \mathrm{dL})] / 405$.

\section{Testosterone Replacement Therapy}

Patients who received TRT (injectable or transdermal) for at least six months were included in the analyses. The injectable regimen was an oil-based injectable blend of four esterized testosterone compounds ( ${ }^{\mathrm{T}}$ Sustanon $250 \mathrm{mg}$; testosterone Propionate of $30 \mathrm{mg}$, testosterone phenylpropionate and testosterone isocaproate of $60 \mathrm{mg}$, and testosterone decanoate of $100 \mathrm{mg}$ ) injected once every 21 days. The transdermal regimen was a once daily testosterone gel formula (testogel $50 \mathrm{mg}$ gel). Laboratory tests in the database were performed using the blood samples collected before the first testosterone administration as the baseline metabolic parameters. 


\begin{tabular}{|c|c|c|c|c|c|}
\hline Variables & $\begin{array}{l}\text { Healthy controls } \\
(n=67)\end{array}$ & $\begin{array}{l}\text { Patients } \\
(n=66)\end{array}$ & $\begin{array}{l}\text { Patients after treatment } \\
(n=66)\end{array}$ & $\mathrm{p} 1^{*}$ & p2* \\
\hline Age, (yr), mean $\pm S D$ & $21.9 \pm 1.3$ & $21.5 \pm 2.0$ & - & 0.24 & - \\
\hline BMI, $\left(\mathrm{kg} / \mathrm{m}^{2}\right)$, mean $\pm S D$ & $23.9 \pm 2.6$ & $23.2 \pm 2.6$ & $24.6 \pm 2.9$ & 0.11 & $<0.001$ \\
\hline WC, $(\mathrm{cm})$, mean $\pm S D$ & $83.7 \pm 7.9$ & $86.8 \pm 9.1$ & $89.1 \pm 8.9$ & 0.04 & 0.001 \\
\hline TG, (mg/dL), mean $\pm S D$ & $80.0(55.0-109.0)$ & $96.0(65.2-132.5)$ & $95.5(72.0-136.0)$ & 0.02 & 0.54 \\
\hline HDL-Chol, (mg/dL), mean $\pm S D$ & $47.3 \pm 8.4$ & $46.7 \pm 9.7$ & $40.7 \pm 9.3$ & 0.71 & $<0.001$ \\
\hline LDL-Chol, (mg/dL), mean士SD & $94.3 \pm 23.2$ & $89.9 \pm 21.9$ & $87.0 \pm 20.3$ & 0.29 & 0.22 \\
\hline FSH, (mIU/mL), mean $\pm S D$ & $3.1 \pm 2.38$ & $0.74 \pm 0.61$ & $0.81 \pm 1.30$ & $<0.001$ & 0.58 \\
\hline LH, (mIU/mL), mean $\pm S D$ & $4.3 \pm 1.58$ & $0.43 \pm 0.54$ & $0.57 \pm 0.93$ & $<0.001$ & 0.05 \\
\hline HOMA-IR ${ }^{\mathrm{a}}$, median (min.-max.) & $1.47(0.95-1.93)$ & $1.95(1.47-2.88)$ & $2.36(1.41-3.30)$ & $<0.001$ & 0.03 \\
\hline Platelet count, $\times 10^{3}$, cells $/ \mu \mathrm{L}$, mean \pm SD & $232.1 \pm 49.2$ & $262.9 \pm 50.7$ & $257.2 \pm 52.2$ & 0.001 & 0.39 \\
\hline MPV, (fL), mean $\pm S D$ & $8.16 \pm 0.92$ & $8.63 \pm 0.87$ & $8.79 \pm 0.91$ & 0.004 & 0.14 \\
\hline PLR, mean $\pm S D$ & $100.59 \pm 25.49$ & $114.01 \pm 25.68$ & $110.86 \pm 37.44$ & 0.003 & 0.45 \\
\hline NLR, mean $\pm S D$ & $1.57 \pm 0.79$ & $1.57 \pm 0.53$ & $1.54 \pm 0.66$ & 0.96 & 0.61 \\
\hline \multicolumn{6}{|c|}{$\begin{array}{l}\text { Data were expressed as mean } \pm \text { standard deviation or median (minimum-maximum). }{ }^{*} \text { Pearson's correlation (p1: between healthy controls and patients; p2: between } \\
\text { before and after testosterone replacement). } \\
\text { BMI: Body mass index, WC: Waist circumference, FBG: Fasting blood glucose, TG: Triglyceride, HDL-Chol: High-density lipoprotein cholesterol, LDL-Chol: Low- } \\
\text { density lipoprotein cholesterol, FSH: Follicle stimulating hormone, LH: Luteinizing hormone, T. Testosterone: Total testosterone, HOMA-IR: Homeostatic model } \\
\text { assessment for insulin resistance, MPV: Mean platelet volume, PLR: Platelet-to-lymphocyte ratio, NLR: Neutrophil-to-lymphocyte ratio, yr: Year }\end{array}$} \\
\hline
\end{tabular}
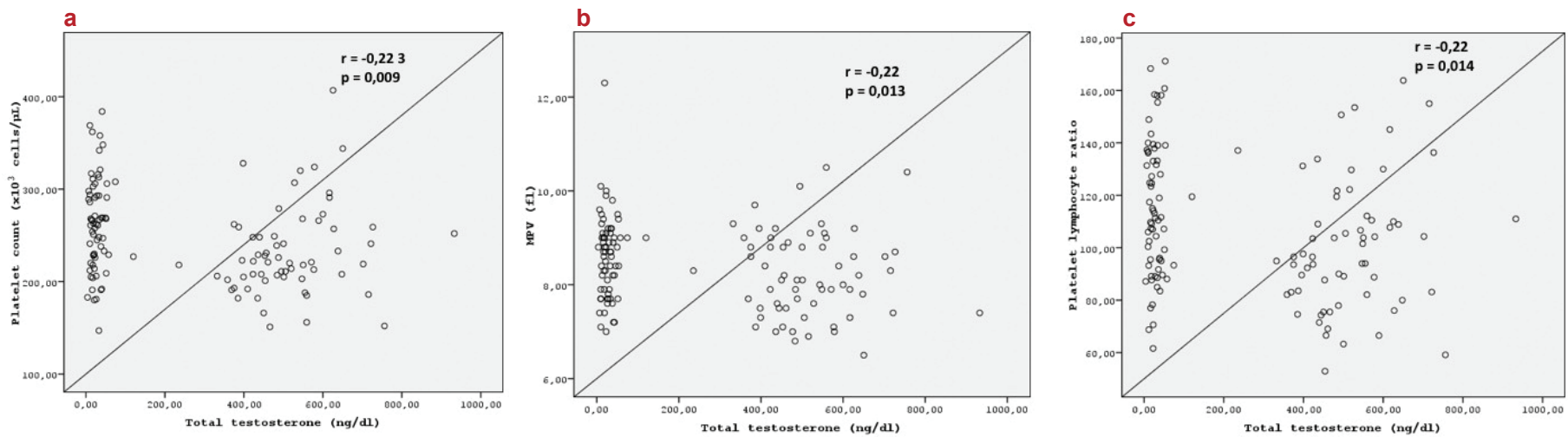

Figure 1. Scatter plot diagrams of correlation between total testosterone and platelet count (a), mean platelet volume (b) and platelet-to-lymphocyte ratio (c)

\section{Statistical Analysis}

The statistical analyses were performed by Statistical Package for the Social Sciences version 18.0 (Chicago, IL, USA). Results were expressed as meantstandard deviation or median (minimum-maximum). The Kolmogorov-Smirnov test was used for normality assessments, and the Levene's test was used to evaluate the equality of variance. While the Student's t-test and Mann-Whitney $U$ test were used for inter-group differences as appropriate, paired samples t-test was used for intra-group changes at two time points. Relationships among MPV level, PLR, NLR, and clinical and biochemical parameters were evaluated by the Pearson correlation coefficient. Stepwise multiple regression analysis was performed to test whether MPV level, PLR, and NLR were independently associated with the total testosterone level. Differences were considered significant at $p<0.05$.

\section{Results}

A total of 66 treatment-naïve young male patients with $\mathrm{CHH}$ (mean age: $21.5 \pm 2.0$ years) and a group of 67 healthy male subjects matched for age and BMI (mean age: $21.9 \pm 1.3$ years) were included. The demographic and biochemical characteristics 


\begin{tabular}{|c|c|c|c|c|c|c|c|c|}
\hline \multirow[t]{2}{*}{ Variables } & \multicolumn{2}{|c|}{ Platelet count } & \multicolumn{2}{|l|}{ MPV } & \multicolumn{2}{|l|}{ PLR } & \multicolumn{2}{|l|}{ NLR } \\
\hline & $r^{*}$ & $p^{*}$ & $r^{*}$ & $p^{*}$ & $r^{*}$ & $\mathbf{p}^{*}$ & $r^{*}$ & $\mathbf{p}^{*}$ \\
\hline WC (cm) & 0.02 & 0.85 & 0.21 & 0.02 & -0.13 & 0.13 & -0.05 & 0.58 \\
\hline BMI $\left(k g / m^{2}\right)$ & -0.07 & 0.46 & 0.10 & 0.23 & -0.21 & 0.02 & -0.14 & 0.12 \\
\hline HDL-Chol (mg/dL) & 0.06 & 0.51 & -0.03 & 0.73 & 0.07 & 0.40 & -0.06 & 0.49 \\
\hline Triglyceride (mg/dL) & 0.05 & 0.60 & -0.002 & 0.98 & -0.07 & 0.43 & 0.04 & 0.67 \\
\hline Insulin ( $\mu \mathrm{U} / \mathrm{mL})$ & 0.11 & 0.21 & -0.18 & 0.04 & 0.06 & 0.54 & -0.01 & 0.91 \\
\hline HOMA-IR & 0.12 & 0.18 & -0.18 & 0.055 & 0.05 & 0.57 & -0.02 & 0.86 \\
\hline FSH (mlU/mL) & -0.15 & 0.10 & -0.29 & 0.001 & -0.24 & 0.008 & -0.09 & 0.32 \\
\hline LH (mIU/mL) & -0.21 & 0.02 & -0.30 & 0.001 & -0.24 & 0.008 & 0.04 & 0.63 \\
\hline Total testosterone (ng/dL) & -0.23 & 0.009 & -0.22 & 0.013 & -0.22 & 0.014 & 0.01 & 0.87 \\
\hline
\end{tabular}

of the patients and control subjects were shown in Table 1. WC $(p=0.04)$, TG $(p=0.02)$, insulin $(p<0.001)$, HOMA-IR $(p<0.001)$, platelet count $(p=0.001), \operatorname{MPV}(p=0.004)$, and PLR $(p=0.003)$ were significantly higher, and $\mathrm{FSH}, \mathrm{LH}$, and total testosterone $(p<0.001)$ level were significantly lower in patients with $\mathrm{CHH}$ than in healthy controls.

After 5.9 \pm 2.1 months of follow-up with TRT, $\mathrm{CHH}$ patients had significantly elevated BMI, WC, total testosterone, and HOMAIR ( $p<0.001, p=0.001, p<0.001$, and $p=0.03$, respectively) and decreased HDL-Chol level $(p<0.001)$; however, changes in platelet count, MPV level, PLR, and NLR were not significant.

Among the $\mathrm{CHH}$ patients, platelet count correlated negatively with $\mathrm{LH}(\mathrm{r}=-0.21, \mathrm{p}=0.02)$ and total testosterone level $(r=-0.23, p=0.009)$ (Figure 1a). MPV level positively correlated with WC $(r=0.21, p=0.019)$ and negatively correlated with insulin $(r=-0.18, p=0.04)$, FSH $(r=-0.29, p=0.01)$, LH $(r=-0.30, p=0.01)$ and total testosterone level $(r=-0.22, p=0.013)$ (Figure 1b). PLR negatively correlated with LH $(r=-0.24, p=0.008), F S H(r=-0.24$, $p=0.008)$, BMI ( $r=-0.21, p=0.02)$, and total testosterone level $(r=-$ $0.22, p=0.014$ ) (Figure 1c) (Table 2).

\section{Discussion}

The results of the present study showed that platelet count, MPV level, and PLR were significantly higher in treatment naïve young patients with $\mathrm{CHH}$. There was also a significant association between these parameters and total testosterone level. In addition, there was no effect of TRT on the platelet count, MPV level, PLR, and NLR. These results may reflect platelet activation in patients with hypogonadism.

The risk of CVD is increased in patients with hypogonadism (16). Patients with type 2 diabetes, metabolic syndrome, and CVD also have low testosterone level $(17,18)$. Therefore, it seems that there is a mutual link between hypogonadism and cardiometabolic diseases. Furthermore, several physiological factors including aging (19), obesity (20), and commonly used medications (21) may also confound the relationship between hypogonadism and cardiometabolic diseases. For this reason, we enrolled the young treatment naïve patients with $\mathrm{CHH}$, who did not have other chronic diseases. In a previous study, we have reported that these patients have metabolic derangements such as visceral obesity and dyslipidemias at a very young age $(15,22)$. Similarly, in the present study, higher WC, TG, and HOMA-IR level in patients with $\mathrm{CHH}$ indicated the metabolic derangements in these patients and supported our previous results. However, the precise mechanism of this increased $\mathrm{CV}$ risk in patients with hypogonadism remains unclear.

Platelets have a crucial role in the emergence of atherosclerosis. It is reported that there is an association between higher platelet count and adverse outcomes (23). MPV is a simple marker of platelet size and reactivity (24) and its increased level is considered to be a CV risk factor $(7,25)$. Larger platelets are metabolically more active than smaller ones and have higher thrombotic potential. Larger platelets contain more granules and higher thromboxane A2 level and also express more glycoprotein $1 b$ and $2 b / 3 a$ receptors. Increased MPV levels are reported in different types of CVD. MPV level is increased in patients with coronary artery disease (7) and are also reported to be associated with the severity of atherosclerosis. Shah et al. (26) also showed that MPV was strongly and independently associated with the presence and severity of diabetes. In addition, several studies have showed that MPV level is increased in all subtypes of ischemic stroke (7). In our study, we also found increased platelet count and MPV level in patients with $\mathrm{CHH}$. In addition, there was also negative correlation between total testosterone level and platelet count and MPV level. These findings imply that patients with $\mathrm{CHH}$ are susceptible to increased platelet activation even at a young 
age. Our results are consistent with the study of Carlioglu et al. (27) that showed elevated MPV level in patients with idiopathic hypogonadotropic hypogonadism. We also showed increased platelet count and metabolic derangements such as increased WC, TG, and HOMA-IR level.

PLR is also considered a new marker of CV risk. Elevated PLR indicates a state of activated hemostasis that leads to adverse CV events $(10,11)$. Similar to platelets, white blood cells also play an important role in the pathogenesis of CVD. It is showed that higher neutrophil counts were related to increased atherosclerosis severity in coronary artery disease. NLR, a new inflammatory biomarker for CVD, is associated with severity and prognosis of CVD (28). This is the first study to evaluate PLR and NLR in patients with $\mathrm{CHH}$. We found that PLR was elevated in patients with $\mathrm{CHH}$, whereas NLR was not different between patients with $\mathrm{CHH}$ and the healthy controls. When PLR is considered to be a CV risk marker, these results may indicate that platelets, but not neutrophils, may play a crucial role in the pathogenesis of increased $\mathrm{CV}$ risk in patients with hypogonadism.

TRT is an emerging treatment option for the metabolic abnormalities in patients with hypogonadism. However, there are controversial data and growing skepticism about the effect of TRT on the improvement of the patient's cardiometabolic risk. Some studies report that TRT improves metabolic parameters $(29,30)$, whereas other studies report increased CV events in patients with hypogonadism under TRT $(31,32)$. However, so far there has been no randomized placebo-controlled prospective study which has investigated the long-term CV effects of TRT in patients with hypogonadism. Therefore, measuring the surrogate marker of CV risk is an alternate way to evaluate the CV effects of TRT in patients with hypogonadism. In the present study, we found no effect of TRT on the whole blood count indices such as platelet count, MPV level, PLR, or NLR, which are surrogate markers of $\mathrm{CV}$ risk. This study showed that patients with $\mathrm{CHH}$ had worse platelet functions than healthy controls. However, approximately six months of TRT do not improve poor platelet function in patients with $\mathrm{CHH}$. Moreover, TRT led to poor metabolic control such as increased BMI, WC, and HOMA-IR level and decreased HDL-Chol level. TRT may ignite pubertal growth in these young patients, which is not seen in elderly hypogonadal patients of etiologies. This may partly explain the rapid increase in BMI and WC and poor metabolic control. Therefore, our results do not support the studies showing that TRT improves CV outcomes $(29,30)$. The short time of TRT or the retrospective nature of our study may be the reason of these conflicting results.

This study may have both limitations and strengths. First, the retrospective design of the study does not allow a causal relationship. Also, the specific population of young treatment naïve patients included in the analyses may not adequately represent all hypogonadism patients. In addition, the small sample size and relatively short duration of follow-up precludes further mechanistic comments. On the other hand, the study population is unique for the fact that it is very uncommon to identify untreated $\mathrm{CHH}$ patients until adult age. The homogeneous study population of young male patients, and the lack of confounding factors such as chronic metabolic disorders and concomitant medications can be counted among the strengths of this study.

\section{Conclusion}

The results of the present study showed that the platelet count and surrogate markers of $\mathrm{CV}$ risk such as MPV level and PLR were significantly increased in patients with $\mathrm{CHH}$. Moreover, the short time TRT in young treatment naïve patients with $\mathrm{CHH}$ showed no effect on the platelet count and size. Future prospective, randomized controlled studies are warranted to clarify the role of platelet functions in the pathogenesis of increased $\mathrm{CV}$ risk in patients with hypogonadism.

\section{Acknowledgement}

We are grateful to İlker Taşçı, M.D., Prof. for critically revising the manuscript.

\section{Ethics}

Ethics Committee Approval: The Local Ethical Committee of University of Health Sciences Turkey Gülhane Faculty of Medicine approved the study (date: 16.06.2016, approval date: 50687569-1491-440-16/1648-1562).

Informed Consent: Retrospective study.

Peer-review: Externally peer-reviewed.

\section{Authorship Contributions}

Design: C.H., O.A., A.S., Data Collection or Processing: I.D., O.D., C.M., Analysis or Interpretation: İ.D., C.H., A.A., Literature Search: I.D., O.A., C.M., A.A., Writing: I.D., C.H., A.S.

Conflict of Interest: No conflict of interest was declared by the authors.

Financial Disclosure: The authors declared that this study received no financial support.

\section{References}

1. Traish AM, Saad F, Feeley RJ, Guay A. The dark side of testosterone deficiency: III. Cardiovascular disease. J Androl. 2009;30:477-494.

2. Kupelian V, Page ST, Araujo AB, Travison TG, Bremner WJ, McKinlay JB. Low sex hormone-binding globulin, total testosterone, and symptomatic androgen deficiency are associated with development of the metabolic syndrome in nonobese men. J Clin Endocrinol Metab. 2006;91:843-850. 
3. Alkamel A, Shafiee A, Jalali A, Boroumand M, Nozari Y. The association between premature coronary artery disease and level of testosterone in young adult males. Arch Iran Med. 2014;17:545-550.

4. Hu X, Rui L, Zhu T, et al. Low testosterone level in middleaged male patients with coronary artery disease. Eur J Intern Med. 2011;22:e133-136.

5. Baetta R, Corsini A. Role of polymorphonuclear neutrophils in atherosclerosis: current state and future perspectives. Atherosclerosis. 2010;210:1-13.

6. Elagizi A, Köhler TS, Lavie CJ. Testosterone and Cardiovascular Health. Mayo Clin Proc. 2018;93:83-100.

7. Vizioli L, Muscari S, Muscari A. The relationship of mean platelet volume with the risk and prognosis of cardiovascular diseases. Int J Clin Pract. 2009;63:1509-1515.

8. Kaya MG, Yarlioglues M, Gunebakmaz O, et al. Platelet activation and inflammatory response in patients with nondipper hypertension. Atherosclerosis. 2010;209:278-282.

9. Hekimsoy Z, Payzin B, Ornek T, Kandoğan G. Mean platelet volume in Type 2 diabetic patients. J Diabetes Complications. 2004;18:173-176.

10. Azab B, Shah N, Akerman M, McGinn JT Jr. Value of platelet/lymphocyte ratio as a predictor of all-cause mortality after non-ST-elevation myocardial infarction. J Thromb Thrombolysis. 2012;34:326-334.

11. Ugur M, Gul M, Bozbay M, et al. The relationship between platelet to lymphocyte ratio and the clinical outcomes in ST elevation myocardial infarction underwent primary coronary intervention. Blood Coagul Fibrinolysis. 2014;25:806-811.

12. Sunbul $M$, Gerin F, Durmus $E$, et al. Neutrophil to lymphocyte and platelet to lymphocyte ratio in patients with dipper versus non-dipper hypertension. Clin Exp Hypertens. 2014;36:217-221.

13. Papa A, Emdin M, Passino C, Michelassi C, Battaglia D, Cocci F. Predictive value of elevated neutrophil-lymphocyte ratio on cardiac mortality in patients with stable coronary artery disease. Clin Chim Acta. 2008;395:27-31.

14. Sonmez A, Haymana C, Aydogdu A, et al. Endothelial dysfunction, insulin resistance and inflammation in congenital hypogonadism, and the effect of testosterone replacement. Endocr J. 2015;62:605-613.

15. Haymana C, Sonmez A, Aydogdu A, et al. Visceral adiposity index and triglyceride/high-density lipoprotein cholesterol ratio in hypogonadism. Arch Endocrinol Metab. 2017;61:282-287.

16. Laughlin GA, Barrett-Connor E, Bergstrom J. Low serum testosterone and mortality in older men. J Clin Endocrinol Metab. 2008;93:68-75.

17. Khaw KT, Dowsett M, Folkerd E, et al. Endogenous testosterone and mortality due to all causes, cardiovascular disease, and cancer in men: European prospective investigation into cancer in Norfolk (EPIC-Norfolk)
Prospective Population Study. Circulation. 2007;116:26942701.

18. Dhindsa S, Miller MG, McWhirter CL, et al. Testosterone concentrations in diabetic and nondiabetic obese men. Diabetes Care. 2010;33:1186-1192.

19. Harman SM, Metter EJ, Tobin JD, Pearson J, Blackman MR; Baltimore Longitudinal Study of Aging. Longitudinal effects of aging on serum total and free testosterone levels in healthy men. Baltimore Longitudinal Study of Aging. J Clin Endocrinol Metab. 2001;86:724-731.

20. Haffner SM. Sex hormones, obesity, fat distribution, type 2 diabetes and insulin resistance: epidemiological and clinical correlation. Int J Obes Relat Metab Disord. 2000;24 Suppl 2:S56-58.

21. Stanworth RD, Kapoor D, Channer KS, Jones TH. Statin therapy is associated with lower total but not bioavailable or free testosterone in men with type 2 diabetes. Diabetes Care. 2009;32:541-546.

22. Sonmez A, Haymana C, Bolu E, et al. Metabolic syndrome and the effect of testosterone treatment in young men with congenital hypogonadotropic hypogonadism. Eur J Endocrinol. 2011;164:759-764.

23. Thaulow E, Erikssen J, Sandvik L, Stormorken H, Cohn PF. Blood platelet count and function are related to total and cardiovascular death in apparently healthy men. Circulation. 1991;84:613-617.

24. Park $\mathrm{Y}$, Schoene N, Harris W. Mean platelet volume as an indicator of platelet activation: methodological issues. Platelets. 2002;13:301-306.

25. Chu SG, Becker RC, Berger PB, et al. Mean platelet volume as a predictor of cardiovascular risk: a systematic review and meta-analysis. J Thromb Haemost. 2010;8:148-156.

26. Shah B, Sha D, Xie D, Mohler ER, Berger JS. The relationship between diabetes, metabolic syndrome, and platelet activity as measured by mean platelet volume: the National Health And Nutrition Examination Survey, 19992004. Diabetes Care. 2012;35:1074-1078.

27. Carlioglu A, Durmaz SA, Kibar YI, Ozturk Y, Tay A. Mean platelet volume in a patient with male hypogonadotropic hypogonadism: the relationship between low testosterone, metabolic syndrome, impaired fasting glucose and cardiovascular risk. Blood Coagul Fibrinolysis. 2015;26:811815.

28. Horne BD, Anderson JL, John JM, et al. Which white blood cell subtypes predict increased cardiovascular risk? J Am Coll Cardiol. 2005;45:1638-1643.

29. Kapoor D, Goodwin E, Channer KS, Jones TH. Testosterone replacement therapy improves insulin resistance, glycaemic control, visceral adiposity and hypercholesterolaemia in hypogonadal men with type 2 diabetes. Eur J Endocrinol. 2006;154:899-906.

30. Li JY, Zhu JC, Dou JT, et al. Effects of androgen supplementation therapy on partial androgen deficiency in 
the aging male: a preliminary study. Aging Male. 2002;5:4751.

31. Basaria S, Coviello AD, Travison TG, et al. Adverse events associated with testosterone administration. $\mathrm{N}$ Engl $\mathrm{J}$ Med. 2010;363:109-122.
32. Vigen $\mathrm{R}$, O'Donnell $\mathrm{Cl}$, Barón $\mathrm{AE}$, et al. Association of testosterone therapy with mortality, myocardial infarction, and stroke in men with low testosterone levels. JAMA. 2013;310:1829-1836. 